\title{
Extended Use of Dabigatran, Warfarin, or Placebo in Venous Thromboembolism
}

\author{
Sam Schulman, M.D., Ph.D., Clive Kearon, M.D., \\ Ajay K. Kakkar, M.B., B.S., Ph.D., Sebastian Schellong, M.D., \\ Henry Eriksson, M.D., Ph.D., David Baanstra, M.Sc., \\ Anne Mathilde Kvamme, M.Sc.Pharm., Jeffrey Friedman, M.D., \\ Patrick Mismetti, M.D., and Samuel Z. Goldhaber, M.D., \\ for the RE-MEDY and the RE-SONATE Trials Investigators*
}

A BSTRACT

BACKGROUND

Dabigatran, which is administered in a fixed dose and does not require laboratory monitoring, may be suitable for extended treatment of venous thromboembolism.

\section{METHODS}

In two double-blind, randomized trials, we compared dabigatran at a dose of $150 \mathrm{mg}$ twice daily with warfarin (active-control study) or with placebo (placebo-control study) in patients with venous thromboembolism who had completed at least 3 initial months of therapy.

\section{RESULTS}

In the active-control study, recurrent venous thromboembolism occurred in 26 of 1430 patients in the dabigatran group $(1.8 \%)$ and 18 of 1426 patients in the warfarin group (1.3\%) (hazard ratio with dabigatran, $1.44 ; 95 \%$ confidence interval [CI], 0.78 to 2.64; $\mathrm{P}=0.01$ for noninferiority). Major bleeding occurred in 13 patients in the dabigatran group $(0.9 \%)$ and 25 patients in the warfarin group (1.8\%) (hazard ratio, 0.52 ; $95 \%$ CI, 0.27 to 1.02). Major or clinically relevant bleeding was less frequent with dabigatran (hazard ratio, $0.54 ; 95 \% \mathrm{CI}, 0.41$ to 0.71 ). Acute coronary syndromes occurred in 13 patients in the dabigatran group $(0.9 \%)$ and 3 patients in the warfarin group $(0.2 \%)(\mathrm{P}=0.02)$. In the placebo-control study, recurrent venous thromboembolism occurred in 3 of 681 patients in the dabigatran group $(0.4 \%)$ and 37 of 662 patients in the placebo group (5.6\%) (hazard ratio, $0.08 ; 95 \% \mathrm{CI}, 0.02$ to $0.25 ; \mathrm{P}<0.001)$. Major bleeding occurred in 2 patients in the dabigatran group $(0.3 \%)$ and 0 patients in the placebo group. Major or clinically relevant bleeding occurred in 36 patients in the dabigatran group (5.3\%) and 12 patients in the placebo group (1.8\%) (hazard ratio, 2.92; 95\% CI, 1.52 to 5.60 ). Acute coronary syndromes occurred in 1 patient each in the dabigatran and placebo groups.

\section{CONCLUSIONS}

Dabigatran was effective in the extended treatment of venous thromboembolism and carried a lower risk of major or clinically relevant bleeding than warfarin but a higher risk than placebo. (Funded by Boehringer Ingelheim; RE-MEDY and RE-SONATE ClinicalTrials.gov numbers, NCT00329238 and NCT00558259, respectively.)
From the Department of Medicine, McMaster University and Thrombosis and Atherosclerosis Research Institute, Hamilton, ON, Canada (S. Schulman, C.K.); the Department of Hematology, Karolinska University Hospital, Stockholm (S. Schulman), and the Department of Medicine, Sahlgrenska University Hospital-Östra, Gothenburg (H.E.) - both in Sweden; the Thrombosis Research Institute and University College London, London (A.K.K.); Medical Division 2, Municipal Hospital Friedrichstadt, Dresden, Germany (S. Schellong); Clinical Research, Boehringer Ingelheim, Alkmaar, the Netherlands (D.B.); Clinical Research, Boehringer Ingelheim, Asker, Norway (A.M.K.); Boehringer Ingelheim, Ridgefield, CT (J.F.); the Department of Vascular Pathology, Bellevue Hospital, Saint Etienne, France (P.M.); and Brigham and Women's Hospital and Harvard Medical School, Boston (S.Z.G.). Address reprint requests to Dr. Schulman at Thrombosis Service, HHS-General Hospital, 237 Barton St. E., Hamilton, ON L8L 2X2, Canada, or at schulms@mcmaster.ca.

* Members of the RE-MEDY and RESONATE study groups are listed in the Supplementary Appendix, available at NEJM.org.

N Engl J Med 2013;368:709-18. DOI: 10.1056/NEJMoal113697

Copyright @ 2013 Massachusetts Medical Society. 
NTICOAGULANT TREATMENT WITH VItamin $\mathrm{K}$ antagonists is recommended for patients with venous thromboembolism. ${ }^{1}$ Most patients receive at least 3 months of treatment. Long-term treatment is recommended if there are risk factors for recurrence, such as multiple thrombotic episodes. ${ }^{1}$ In the absence of clear contraindications to anticoagulant therapy, the risk of major bleeding is approximately $1 \%$ per year with extended vitamin $\mathrm{K}$ antagonist therapy after venous thromboembolism. ${ }^{2}$ The risk of major bleeding, together with the need for frequent laboratory monitoring and dose adjustments, makes long-term treatment problematic.

Dabigatran, a direct thrombin inhibitor, does not require frequent monitoring and dose adjustments. At a dose of $150 \mathrm{mg}$ twice daily, it was shown in one trial to be noninferior to warfarin for the initial 6-month treatment of venous thromboembolism and was associated with a lower rate of clinically relevant nonmajor bleeding. ${ }^{3}$ These findings suggested that dabigatran might also be suitable for extended therapy beyond the initial period of treatment. We performed two studies to evaluate the efficacy and safety of dabigatran during long-term prophylaxis after venous thromboembolism. In one trial, dabigatran was compared with warfarin (the active-control study); in the other trial, dabigatran was compared with placebo (the placebo-control study).

\section{METHODS}

\section{STUDY DESIGN}

The active-control study was designated RE-MEDY, and the placebo-control study was designated RE-SONATE. Both studies had a randomized, double-blind design and enrolled patients who had completed at least 3 months of treatment for venous thromboembolism. Both studies were funded, designed, and conducted, and the data analyzed, by Boehringer Ingelheim in conjunction with the steering committee, which was a common steering committee for the two trials. The members of the steering committee (the seven academic authors), who had independent access to the data, wrote the manuscript and made the decision to submit it for publication. The members of the steering committee vouch for the accuracy and completeness of the data and the analyses and for the fidelity of this report to the trial protocols, which are available with the full text of this article at NEJM.org.

\section{STUDY PATIENTS}

We recruited patients for the active-control study from 265 sites in 33 countries and for the placebocontrol study from 147 sites in 21 countries. Patients were eligible if they were at least 18 years of age and had objectively confirmed, symptomatic, proximal deep-vein thrombosis or pulmonary embolism that had already been treated with an approved anticoagulant or if they had received dabigatran in one of two previous clinical trials of short-term treatment of venous thromboembolism: the RE-COVER ${ }^{3}$ and RE-COVER $\mathrm{II}^{4}$ studies.

The main difference between the two trials in the selection of patients was that participants in the active-control study were considered to be at increased risk for recurrent venous thromboembolism on the basis of the site investigator's assessment. The required duration of initial treatment before trial enrollment was 3 to 12 months for the active-control study and 6 to 18 months for the placebo-control study. Full lists of inclusion and exclusion criteria for each trial are provided in the Supplementary Appendix, available at NEJM.org.

All patients provided written informed consent. The institutional review board at each participating clinical center approved the studies.

\section{RANDOMIZATION AND TREATMENT}

In both studies, patients underwent randomization by means of an interactive voice-response system. Randomization was stratified according to the presence or absence of active cancer and according to the index diagnosis (deep-vein thrombosis or pulmonary embolism) in the active-control study and according to study center in the placebo-control study. Prior anticoagulant therapy was discontinued, and the study drug was started when the international normalized ratio (INR) was 2.3 or lower. If the patient was enrolled from the RE-COVER study or the RE-COVER II study, a point-of-care coagulometer with encrypted INR results was used to guide the transition so that the patients and investigators would remain unaware of the initial treatment.

In the active-control study, patients were assigned in a 1:1 ratio to receive active dabigatran (at a fixed dose of $150 \mathrm{mg}$ twice daily) and a warfarin-like placebo or active warfarin and a dabigatran-like placebo. Bilateral compression ultrasonography of the leg veins was performed within 72 hours after randomization. We adjusted the dose of warfarin or placebo to maintain an INR of 2.0 to 3.0 with the use of a point- 
of-care instrument that provided an encrypted INR. The true or sham INR was then obtained by means of an interactive voice-response system with a central computer that had been programmed with the randomization schedule.

In the placebo-control study, patients were assigned in a 1:1 ratio to receive dabigatran (at a fixed dose of $150 \mathrm{mg}$ twice daily) or a matching placebo. Laboratory monitoring of coagulation was not performed in the placebo-control study.

\section{FOLLOW-UP AND OUTCOME MEASURES}

Patients were assessed at 15 and 30 days after randomization, then monthly until day 180 . After that, patients in the active-control study were assessed every 90 days until the end of treatment; INR or sham INR results were obtained at intervals of no longer than 4 weeks. An additional visit occurred 30 days after the end of treatment in both studies.

The active-control study was initially designed for 18 months of treatment. Because of a lowerthan-projected event rate, the protocol was amended to increase the sample and extend the planned treatment period for patients already enrolled who consented to the extension, with the resulting planned study treatment period ranging from 6 to 36 months. The placebo-control study was amended, 6 months after recruitment of the first patient, to extend the follow-up to 12 months after completion of the study treatment to evaluate the long-term risk of recurrence.

In both studies, the primary efficacy outcome was recurrent symptomatic and objectively verified venous thromboembolism or death associated with venous thromboembolism (or unexplained death in the placebo-control study). Safety outcomes included major bleeding and clinically relevant nonmajor bleeding. Definitions of the efficacy and safety outcomes are provided in the Supplementary Appendix. Central committees, whose members were not aware of the treatment assignments, adjudicated suspected cases of recurrent venous thromboembolism, bleeding, death, acute coronary events (as well as cerebrovascular events in the placebo-control study), and liverfunction abnormalities (according to clinical and routine laboratory data).

\section{STATISTICAL ANALYSIS}

The active-control study was designed to show that dabigatran is noninferior to warfarin in preventing recurrent venous thromboembolism (including death related to venous thromboembo- lism). The sample size was determined on the basis of an expected rate of the primary efficacy outcome of $2.0 \%$ in both groups, ${ }^{5-8}$ with a power of $85 \%$ to exclude a hazard ratio of 2.85 (the noninferiority margin for the hazard ratio), $, 3,9-14$ and an absolute increase in the risk of recurrent venous thromboembolism of 2.8 percentage points at 18 months (the noninferiority margin for the risk difference), at a one-sided alpha level of 0.025 . To meet these specifications, we estimated that we would need to enroll 2000 patients. In the protocol amendment described above, the sample was increased to 2850 patients. Details of the sample-size calculation and the determination of the noninferiority margin are provided in the Supplementary Appendix.

The amended protocol for the active-control study resulted in a study population with a treatment duration ranging from 6 to 36 months. Consequently, the protocol was further revised to group the study participants into three cohorts according to the duration of treatment ( $<18$ months, 18 months, or $>18$ months) and to perform a meta-analysis of these three cohorts for the primary efficacy outcome. Details of this analytic method are provided in the Supplementary Appendix. The prespecified analyses were the hazard ratio for the planned treatment period and the risk difference at 18 months. Both estimates had to satisfy the noninferiority criteria for dabigatran to be judged to be noninferior.

The placebo-control study was designed to determine whether dabigatran was superior to placebo for preventing recurrent venous thromboembolism. We assumed a $70 \%$ reduction in the relative risk of the primary outcome with dabigatran ${ }^{15}$ and planned for a power of $95 \%$ to detect this difference, at a two-sided alpha of 0.05 ; on the basis of these specifications, 36 events were required. With an assumed frequency of the primary outcome of 3\% at 6 months in the placebo group, a sample of approximately 1800 patients was needed. The primary efficacy outcome was analyzed with the use of a Cox proportional-hazards model.

In both trials, a modified intention-to-treat analysis was performed for efficacy, with exclusion of patients who did not receive any dose of the study drug. In the safety analyses, we included all events during the period from receipt of the first dose of the study drug until 3 days after receipt of the last dose. All safety analyses and secondary efficacy analyses were prespecified. 


\section{RESULTS}

\section{PATIENTS}

From July 2006 through July 2010, a total of 2866 patients underwent randomization in the activecontrol study, and from November 2007 through September 2010, a total of 1353 patients underwent randomization in the placebo-control study (Fig. S1A and S1B in the Supplementary Appendix). Demographic and clinical characteristics of the participants in both trials are shown in Table 1.

\section{TREATMENT AND FOLLOW-UP}

In the active-control study, one patient in each group mistakenly received the study drug assigned to the other group throughout the study. In the warfarin group, the INR was in the therapeutic range (2.0 to 3.0) for a median of $65.3 \%$ of the time. The INR was below the therapeutic range $17.3 \%$ of the time and above the therapeutic range $12.2 \%$ of the time. Additional characteristics of the treatments are provided in Table 1.
In the active-control study, the study drug was stopped early in 276 patients (19.3\%) in the dabigatran group (in 147 because of an adverse event, 23 because of nonadherence, 2 because of loss to follow-up, 64 because of their decision to stop taking the study medication, and 40 for other reasons) and in 281 patients $(19.7 \%)$ in the warfarin group (in 129 because of an adverse event, 34 because of nonadherence, 6 because of loss to followup, 58 because of their decision to stop taking the study medication, and 54 for other reasons).

In the placebo-control study, 3 patients assigned to placebo mistakenly received dabigatran throughout the study. The study drug was stopped early in 71 patients (10.4\%) in the dabigatran group (in 50 because of an adverse event, 9 because of nonadherence, and 12 because of their decision to stop taking the study medication) and in 99 patients (15.0\%) in the placebo group (in 81 because of an adverse event, 5 because of nonadherence, and 13 because of their decision to stop taking the study medication).

\begin{tabular}{|c|c|c|c|c|}
\hline \multirow[t]{2}{*}{ Characteristic } & \multicolumn{2}{|c|}{ Active-Control Study } & \multicolumn{2}{|c|}{ Placebo-Control Study } \\
\hline & $\begin{array}{c}\text { Dabigatran } \\
(\mathrm{N}=1430)\end{array}$ & $\begin{array}{l}\text { Warfarin } \\
(\mathrm{N}=1426)\end{array}$ & $\begin{array}{c}\text { Dabigatran } \\
(N=681)\end{array}$ & $\begin{array}{c}\text { Placebo } \\
(\mathrm{N}=662)\end{array}$ \\
\hline \multicolumn{5}{|l|}{ Age $-y r$} \\
\hline Mean & $55.4 \pm 15.0$ & $53.9 \pm 15.3$ & $56.1 \pm 15.5$ & $55.5 \pm 15.1$ \\
\hline Range & $18-92$ & $18-93$ & $18-89$ & $18-91$ \\
\hline Female sex - no. (\%) & $559(39.1)$ & $555(38.9)$ & $300(44.1)$ & $298(45.0)$ \\
\hline \multicolumn{5}{|l|}{ Race or ethnic group — no. (\%)† } \\
\hline White & $1288(90.1)$ & $1284(90.0)$ & $610(89.6)$ & $585(88.4)$ \\
\hline Black & $29(2.0)$ & $28(2.0)$ & $9(1.3)$ & $14(2.1)$ \\
\hline Asian & $113(7.9)$ & $114(8.0)$ & $58(8.5)$ & $60(9.1)$ \\
\hline American Indian & 0 & 0 & $4(0.6)$ & $3(0.5)$ \\
\hline \multicolumn{5}{|l|}{ Weight — kg } \\
\hline Mean & $86.1 \pm 19.3$ & $86.0 \pm 18.9$ & $83.7 \pm 18.0$ & $84.0 \pm 18.6$ \\
\hline Range & $40-188$ & $41-182$ & $40-151$ & $40-206$ \\
\hline Estimated creatinine clearance $-\mathrm{ml} / \mathrm{min} \downarrow$ & $104.2 \pm 38.6$ & $106.6 \pm 37.9$ & $99.6 \pm 35.8$ & $101.2 \pm 37.1$ \\
\hline \multicolumn{5}{|l|}{ Type of index event — no. (\%) } \\
\hline Deep-vein thrombosis only & $938(65.6)$ & $922(64.7)$ & $431(63.3)$ & $441(66.6)$ \\
\hline Pulmonary embolism only & $324(22.7)$ & $335(23.5)$ & $183(26.9)$ & $178(26.9)$ \\
\hline Both deep-vein thrombosis and pulmonary embolism & $167(11.7)$ & $168(11.8)$ & $47(6.9)$ & $35(5.3)$ \\
\hline Neither deep-vein thrombosis nor pulmonary embolism $\mathbb{S}$ & $1(0.1)$ & $1(0.1)$ & $20(2.9)$ & $8(1.2)$ \\
\hline \multicolumn{5}{|l|}{ Immobilization — no. (\%) } \\
\hline Transient & $91(6.4)$ & $93(6.5)$ & $49(7.2)$ & $36(5.4)$ \\
\hline Permanent & $3(0.2)$ & $12(0.8)$ & $4(0.6)$ & 0 \\
\hline
\end{tabular}




\begin{tabular}{|c|c|c|c|c|}
\hline \multicolumn{5}{|l|}{ Table 1. (Continued.) } \\
\hline \multirow[t]{2}{*}{ Characteristic } & \multicolumn{2}{|c|}{ Active-Control Study } & \multicolumn{2}{|c|}{ Placebo-Control Study } \\
\hline & $\begin{array}{c}\text { Dabigatran } \\
(\mathrm{N}=1430)\end{array}$ & $\begin{array}{l}\text { Warfarin } \\
(\mathrm{N}=1426)\end{array}$ & $\begin{array}{c}\text { Dabigatran } \\
(\mathrm{N}=681)\end{array}$ & $\begin{array}{c}\text { Placebo } \\
(\mathrm{N}=662)\end{array}$ \\
\hline Known thrombophilia - no. (\%) & $262(18.3)$ & $263(18.4)$ & $87(12.8)$ & $68(10.3)$ \\
\hline Active cancer - no. (\%) & $60(4.2)$ & $59(4.1)$ & 19 & 29 \\
\hline Previous cancer — no. (\%) & NA & NA & $44(6.5)$ & $37(5.6)$ \\
\hline Coronary artery disease - no. (\%) & $120(8.4)$ & $87(6.1) \|$ & $43(6.3)$ & $38(5.7)$ \\
\hline Diabetes mellitus — no. (\%) & $150(10.5)$ & $108(7.6) * *$ & $57(8.4)$ & $50(7.6)$ \\
\hline Hypertension - no. (\%) & $582(40.7)$ & $520(36.5) \|$ & $281(41.3)$ & $240(36.3)$ \\
\hline Treatment duration before randomization - days & $198 \pm 157$ & $200 \pm 117$ & $293 \pm 107$ & $299 \pm 110$ \\
\hline \multicolumn{5}{|l|}{ Enrolled from RE-COVER study — no. (\%) } \\
\hline Dabigatran group & $236(16.5)$ & $254(17.8)$ & $7(1.0)$ & $8(1.2)$ \\
\hline Warfarin group & $283(19.8)$ & $243(17.0)$ & $8(1.2)$ & $4(0.6)$ \\
\hline Enrolled from RE-COVER II study - no. (\%) & $70(4.9)$ & $55(3.9)$ & NA & NA \\
\hline Exposure to study drug — days & $473 \pm 211$ & $474 \pm 206$ & $165 \pm 45$ & $162 \pm 47$ \\
\hline Adherence to study regimen — no. (\%) †† & $1386(98.0)$ & $1387(98.2)$ & $657(96.5)$ & $639(96.5)$ \\
\hline \multicolumn{5}{|c|}{$\begin{array}{l}\text { Plus-minus values are means } \pm \text { SD. All variables in each study were compared between the two groups. There were } \\
\text { no significant differences between groups unless otherwise indicated. NA denotes not applicable. } \\
\text { Race or ethnic group was determined by the investigator. } \\
\text { Creatinine clearance was estimated according to the Cockcroft-Gault method. } \\
\text { In these cases, the initial diagnosis of proximal deep-vein thrombosis or pulmonary embolism was made locally but } \\
\text { could not be confirmed by the central adjudication committee. } \\
\text { Active cancer was an exclusion criterion, and the numbers represent protocol violations. } \\
\mathrm{P}=0.02 \text { for the difference between the dabigatran group and the warfarin group, calculated with the chi-square test. } \\
\mathrm{P}=0.007 \text { for the difference between the dabigatran group and the warfarin group, calculated with the chi-square test. } \\
\text { Adherence was assumed if a pill count for dabigatran or the dabigatran-like placebo indicated an intake of } 80 \text { to } 120 \% \\
\text { of the prescribed dose. In the active-control study, this information was available for } 1415 \text { patients in the dabigatran } \\
\text { group and } 1412 \text { patients in the warfarin group. }\end{array}$} \\
\hline
\end{tabular}

\section{EFFICACY AND SAFETY IN THE ACTIVE-CONTROL STUDY}

The primary outcome for efficacy was confirmed by central adjudication in 26 patients $(1.8 \%)$ in the dabigatran group and in 18 patients (1.3\%) in the warfarin group (Table 2). The overall hazard ratio with dabigatran for time to the first primaryoutcome event was 1.44 (95\% confidence interval [CI], 0.78 to 2.64), and the risk difference at 18 months was 0.38 percentage points $(95 \% \mathrm{CI},-0.50$ to 1.25) (Fig. 1A), calculated with the use of the meta-analytic approach. In a Cox regression analysis of pooled individual data, the hazard ratio was 1.47 ( $95 \%$ CI, 0.80 to 2.68). Dabigatran thus met the criteria for noninferiority to warfarin with regard to the prevention of recurrent or fatal venous thromboembolism $(\mathrm{P}<0.001$ for the risk-difference criterion and $\mathrm{P}=0.01$ for the hazardratio criterion). There were no significant differences in efficacy according to study treatment in predefined subgroups (Fig. S2 in the Supplementary Appendix).

Thirteen patients $(0.9 \%)$ in the dabigatran group had a major bleeding event, as compared with 25 patients $(1.8 \%)$ in the warfarin group. The overall hazard ratio for time to first major bleeding event was 0.52 (95\% CI, 0.27 to 1.02) (Table 2). The sites of major bleeding are presented in Table S1 in the Supplementary Appendix. In both groups, 2 patients had bleeding at two sites. Major or clinically relevant bleeding occurred in 80 patients $(5.6 \%)$ in the dabigatran group and 145 patients $(10.2 \%)$ in the warfarin group (hazard ratio, 0.54 ; $95 \%$ CI, 0.41 to 0.71 ; $\mathrm{P}<0.001)$. The risk of any bleeding is shown in Figure 2A. There were no significant differences in the risk of bleeding according to study treatment in predefined subgroups.

We observed a higher incidence of acute coronary events in the dabigatran group (events in 


\begin{tabular}{|c|c|c|c|c|}
\hline Outcome & $\begin{array}{l}\text { Dabigatran } \\
(\mathrm{N}=1430)\end{array}$ & $\begin{array}{l}\text { Warfarin } \\
(\mathrm{N}=1426)\end{array}$ & $\begin{array}{l}\text { Hazard Ratio } \\
\text { (95\% CI) }\end{array}$ & P Value \\
\hline \multicolumn{5}{|c|}{ no. of patients (\%) } \\
\hline \multicolumn{5}{|l|}{ Efficacy analysis'̀ } \\
\hline $\begin{array}{l}\text { Primary end point of recurrent or fatal venous thrombo- } \\
\text { embolism }\end{array}$ & $26(1.8)$ & $18(1.3)$ & $1.44(0.78-2.64)$ & $0.01 \%$ \\
\hline \multicolumn{5}{|l|}{ Secondary end points } \\
\hline Symptomatic deep-vein thrombosis & $17(1.2)$ & $13(0.9)$ & $1.32(0.64-2.71)$ & 0.46 \\
\hline Symptomatic nonfatal pulmonary embolism & $10(0.7)$ & $5(0.4)$ & $2.04(0.70-5.98)$ & 0.19 \\
\hline Death related to venous thromboembolism & $1(0.1)$ & $1(0.1)$ & $1.01(0.06-16.2)$ & 0.99 \\
\hline All deaths & $17(1.2)$ & $19(1.3)$ & $0.90(0.47-1.72)$ & 0.74 \\
\hline \multicolumn{5}{|l|}{ Safety analysis $\widehat{\jmath}$} \\
\hline Major bleeding event $\emptyset$ & $13(0.9)$ & $25(1.8)$ & $0.52(0.27-1.02)$ & 0.06 \\
\hline Fatal event & 0 & 1 & & \\
\hline Bleeding into critical organ & 8 & 13 & & \\
\hline $\begin{array}{l}\text { Event resulting in fall in hemoglobin level or need } \\
\text { for blood transfusions }\end{array}$ & 9 & 18 & & \\
\hline Major or clinically relevant bleeding event & $80(5.6)$ & $145(10.2)$ & $0.54(0.41-0.71)$ & $<0.001$ \\
\hline Any bleeding event & $277(19.4)$ & $373(26.2)$ & $0.71(0.61-0.83)$ & $<0.001$ \\
\hline Adverse event & $1029(72.0)$ & $1010(70.8)$ & & 0.53 \\
\hline Adverse event leading to discontinuation of study drug & $145(10.1)$ & $126(8.8)$ & & 0.26 \\
\hline Serious adverse event & $227(15.9)$ & $224(15.7)$ & & 0.97 \\
\hline \multicolumn{5}{|l|}{ Acute coronary syndrome } \\
\hline During treatment & $13(0.9)$ & $3(0.2)$ & & 0.02 \\
\hline Within 30 days after treatment & $1(0.1)$ & $3(0.2)$ & & \\
\hline $\mathrm{ALT}>3 \times \mathrm{ULN}$ & $24(1.7)$ & $26(1.8)$ & & \\
\hline ALT $>3 \times$ ULN and bilirubin $>2 \times$ ULN & $2(0.1)$ & $1(0.1)$ & & \\
\hline
\end{tabular}

* ALT denotes alanine aminotransferase, Cl confidence interval, and ULN upper limit of the normal range.

$\dagger$ The efficacy analysis included all randomly assigned patients who received at least one dose of the study drug and was based on data for the planned treatment period, regardless of whether the study drug was discontinued early.

$t$ The $P$ value for the primary outcome is for noninferiority, for which the margin was 2.85 .

$\int$ The safety analysis by default was based on the treatment period and a 3-day washout period after the end of treatment.

I One major bleeding event could be attributed to several of the listed criteria. The sites of the major bleeding events are presented in Table S1 in the Supplementary Appendix.

13 patients [0.9\%]: 10 with myocardial infarction and 3 with unstable angina) than in the warfarin group (events in 3 patients [0.2\%]: 1 with myocardial infarction and 2 with unstable angina). All these events were adjudicated as definite except for myocardial infarction in 1 patient in the dabigatran group and unstable angina in 1 patient in the warfarin group. In each group, 1 patient had an event within 3 days after stopping the study treatment.

\section{EFFICACY AND SAFETY IN THE PLACEBO-CONTROL} STUDY

The primary outcome for efficacy was confirmed by central adjudication in 3 patients $(0.4 \%)$ in the dabigatran group and in 37 patients (5.6\%) in the placebo group. The hazard ratio with dabigatran for time to the first primary-outcome event was 0.08 (95\% CI, 0.02 to $0.25 ; \mathrm{P}<0.001$ ) (Table 3 and Fig. 1B). There were no significant differences in efficacy according to study treatment in predefined subgroups (Fig. S3 in the Supplementary Appendix). The extended 12-month follow-up was completed for 1323 of the 1343 patients who underwent randomization and received the study drug (98.5\%), at which point the cumulative incidence of the primary efficacy outcome was $6.9 \%$ in the dabigatran group as compared with $10.7 \%$ in the placebo group (hazard ratio, 0.61; $95 \% \mathrm{CI}$, 0.42 to 0.88 ). 


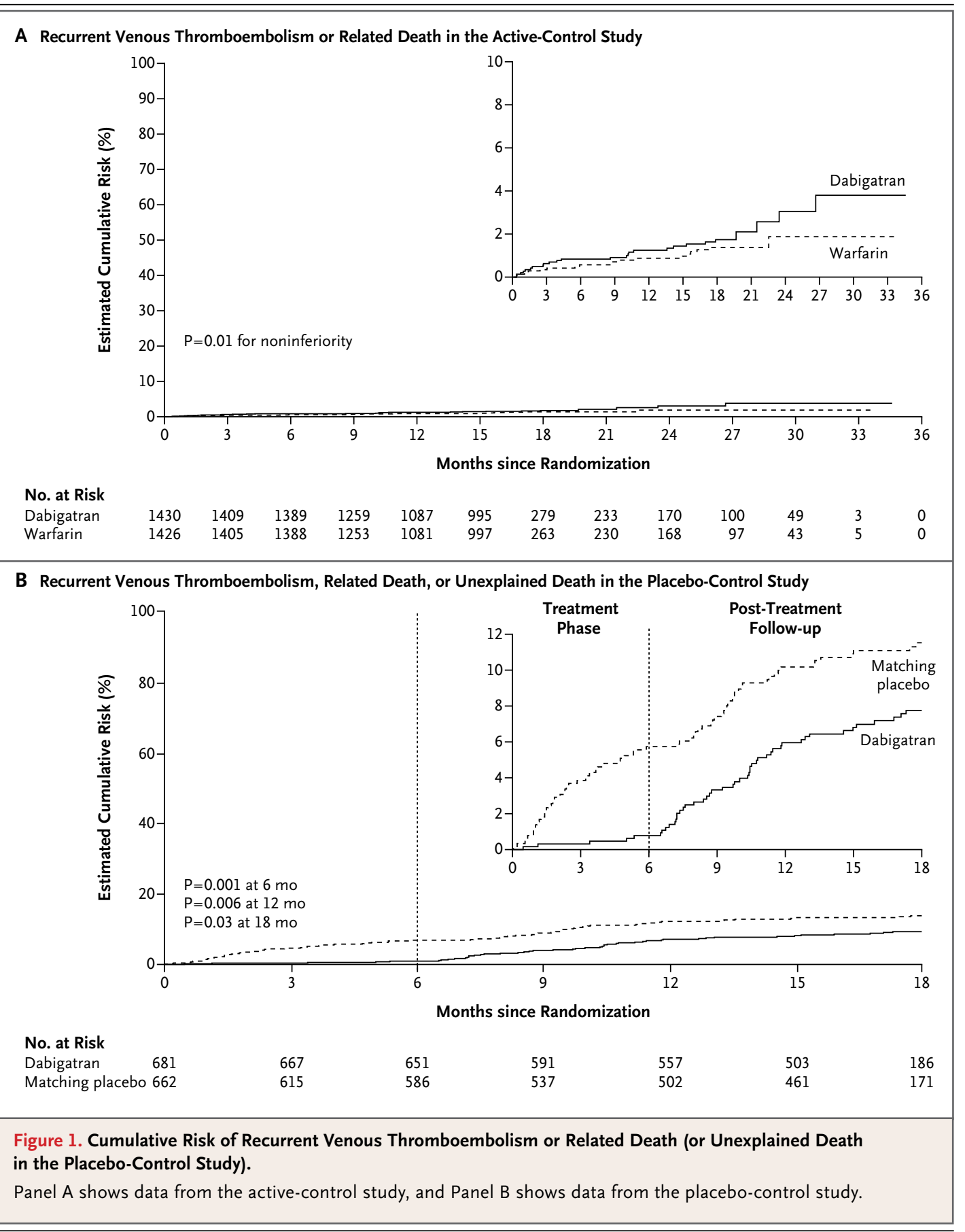

There were 2 patients with major bleeding events, both in the dabigatran group; both events were gastrointestinal bleeding requiring at least 2 units of blood but without a fall in the hemoglobin level of $20 \mathrm{~g}$ per liter or more. Major or clinically relevant nonmajor bleeding occurred in 36 patients (5.3\%) in the dabigatran group as compared with 12 patients $(1.8 \%)$ in the placebo group (hazard ratio, 2.92; 95\% CI, 1.52 to 5.60; $\mathrm{P}=0.001)$. The risk of any bleeding is shown in Figure 2B. Rectal bleeding occurred in 19 patients $(2.8 \%)$ in the dabigatran group and in 5 patients $(0.8 \%)$ in the placebo group, whereas the incidence of bleeding at other sites was similar in the two groups. In this study, there was 1 acute coronary event in each group. 


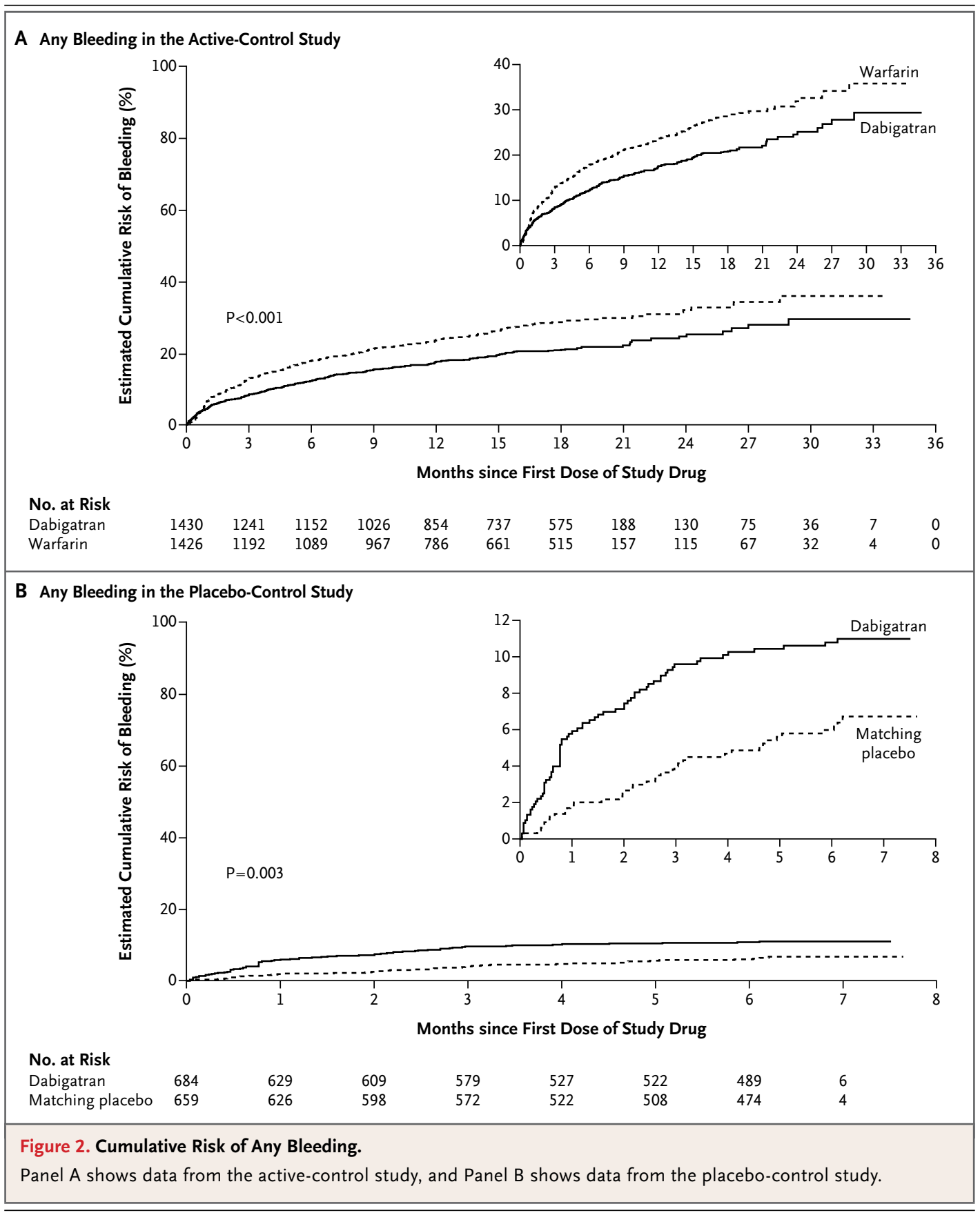

\section{DISCUSSION}

We evaluated the use of dabigatran for the longterm treatment of venous thromboembolism in two complementary randomized trials. In the active-control study, dabigatran met the predefined noninferiority criteria in comparison with warfarin for the prevention of recurrent venous thromboembolism, with fewer major bleed- ing events and significantly fewer clinically relevant nonmajor bleeding events. As compared with placebo, dabigatran significantly reduced the rate of recurrent venous thromboembolism, but with a significantly higher risk of major or clinically relevant nonmajor bleeding.

In the active-control study, there was a numerical increase in recurrent or fatal venous thromboembolic events during treatment with 


\begin{tabular}{|c|c|c|c|c|}
\hline Outcome & $\begin{array}{l}\text { Dabigatran } \\
(\mathrm{N}=681)\end{array}$ & $\begin{array}{l}\text { Placebo } \\
(\mathrm{N}=662)\end{array}$ & $\begin{array}{l}\text { Hazard Ratio } \\
\text { (95\% CI) }\end{array}$ & P Value \\
\hline \multicolumn{5}{|c|}{ no. of patients (\%) } \\
\hline \multicolumn{5}{|l|}{ Efficacy analysis* } \\
\hline $\begin{array}{l}\text { Primary end point of recurrent or fatal venous thrombo- } \\
\text { embolism or unexplained death }\end{array}$ & $3(0.4)$ & $37(5.6)$ & $0.08(0.02-0.25)$ & $<0.001$ \\
\hline \multicolumn{5}{|l|}{ Secondary end points } \\
\hline Symptomatic deep-vein thrombosis & $2(0.3)$ & $22(3.3) \dagger$ & & \\
\hline Symptomatic nonfatal pulmonary embolism & $1(0.1)$ & $14(2.1) \dagger$ & & \\
\hline Unexplained death & 0 & $2(0.3) \ddagger$ & & \\
\hline \multicolumn{5}{|l|}{ Safety analysis $\widehat{\int}$} \\
\hline Major bleeding event $q$ & $2(0.3)$ & 0 & Not estimable & 1.0 \\
\hline $\begin{array}{l}\text { Event resulting in fall in hemoglobin level or need } \\
\text { for blood transfusions }\end{array}$ & 2 & 0 & & \\
\hline Major or clinically relevant bleeding event & $36(5.3)$ & $12(1.8)$ & $2.92(1.52-5.60)$ & 0.001 \\
\hline Any bleeding event & $72(10.5)$ & $39(5.9)$ & $1.82(1.23-2.68)$ & 0.003 \\
\hline Adverse event & $346(50.6)$ & $324(49.2)$ & & \\
\hline Adverse event leading to discontinuation of study drug & $50(7.3)$ & $81(12.3)$ & & \\
\hline Serious adverse event & $47(6.9)$ & $60(9.1)$ & & \\
\hline Acute coronary syndrome during treatment $\|$ & $1(0.1)$ & $1(0.2)$ & & \\
\hline Cerebrovascular event during treatment*** & $2(0.3)$ & $1(0.2)$ & & \\
\hline $\mathrm{ALT}>3 \times \mathrm{ULN}+T$ & $4(0.6)$ & $4(0.6)$ & & \\
\hline
\end{tabular}

* The efficacy analysis included all randomly assigned patients who received at least one dose of the study drug and was based on data for the planned treatment period, regardless of whether the study drug was discontinued early.

$\dagger$ One patient in the placebo group had deep-vein thrombosis and a pulmonary embolism on the same day, and each was considered to be a first event.

$\ddagger$ One of the two deaths occurred when the patient was not receiving the study drug. There were no cases of objectively verified fatal pulmonary embolism or of any other deaths.

$\int$ The safety analysis included 684 patients who received dabigatran and 659 who received placebo. Three patients in the placebo group mistakenly received dabigatran throughout the study.

I None of the major bleeding events were fatal or occurred in a critical organ (see Table S1 in the Supplementary Appendix).

|| There was one myocardial infarction with ST-segment elevation in the dabigatran group and one myocardial infarction without ST-segment elevation in the placebo group.

$* *$ There were two patients with transient ischemic attacks in the dabigatran group and one patient with ischemic stroke in the placebo group.

†† None of the patients had concurrent elevation of the bilirubin level of more than two times the upper limit of the normal range.

dabigatran (26 events [1.8\%], vs. 18 events with warfarin [1.3\%]). The upper limit of the $95 \%$ confidence interval for the hazard ratio (2.64) was close to the predefined noninferiority margin (2.85), and the confidence interval gives boundaries for the event rate with dabigatran as low as $1.0 \%$ and as high as $3.4 \%$. This corresponds to an annual risk of $0.8 \%$ to $2.6 \%$. The annualized point estimates of $1.0 \%$ for warfarin and $1.4 \%$ for dabigatran can be compared with the annualized point estimates in previous trials of extended treatment with warfarin (range,
$0.7 \%$ to $5.0 \%)^{5,6,16-18}$ or with experimental drugs (ximelagatran, 1.3\%; low-dose warfarin, $1.9 \%$ to $2.6 \%$; and rivaroxaban, $1.9 \%$ ). ${ }^{6-9}$ The prespecified noninferiority margin for the hazard ratio of 2.85 is large, since it allows an increase in risk by a factor of nearly 3 to be accepted as noninferior. This is a limitation of the trial design. However, large noninferiority margins have been prespecified in trials of short-term treatment of venous thromboembolism with dabigatran (2.75), ${ }^{5,6,16-18}$ rivaroxaban (2.0),,$^{5,6,16-18}$ and idraparinux (2.0). ${ }^{5,6,16-18}$ 
The efficacy of dabigatran in comparison with placebo (hazard ratio, 0.08 ; 95\% CI, 0.02 to 0.25 ) is of the same magnitude as the efficacy of rivaroxaban versus placebo (hazard ratio, 0.18 ; $95 \%$ CI, 0.09 to 0.39$)^{9}$ and of warfarin versus placebo or control (odds ratio, 0.05 and 0.11 , respectively). ${ }^{5,18}$ The benefit of extended treatment with dabigatran was maintained during the 12 months of extended follow-up after discontinuation of the study drug. The risk of clinically relevant bleeding with dabigatran as compared with placebo (hazard ratio, 2.9; 95\% CI, 1.5 to 5.6) is similar to that of rivaroxaban as compared with placebo (hazard ratio, 5.2; 95\% CI, 2.3 to 11.7 ). ${ }^{9}$

There was a higher rate of acute coronary events with dabigatran than with warfarin, with no significant difference in these events between dabigatran and placebo. In the Randomized Evaluation of Long-Term Anticoagulation Therapy (RE-LY), which compared dabigatran and warfarin in patients with atrial fibrillation, there was a higher risk of acute coronary syndromes with dabigatran, ${ }^{19}$ although after further analysis of the data this difference was no longer significant. ${ }^{20,21}$ However, a recent meta-analysis of seven noninferiority trials showed a significantly higher risk of myocardial infarction or acute coronary syndromes with dabigatran than with the comparator. ${ }^{22}$ Whether dabigatran increases the risk of myocardial infarction is therefore still unclear.

In summary, we evaluated the use of dabigatran for the long-term treatment of venous thromboembolism in two complementary randomized trials. In the active-control study, dabigatran was noninferior to warfarin for the prevention of recurrent venous thromboembolism, with a lower risk of bleeding. In the placebo-control study, dabigatran significantly reduced the rate of recurrent venous thromboembolism, but with a significantly higher risk of bleeding.

Disclosure forms provided by the authors are available with the full text of this article at NEJM.org.

We thank Dr. Harry Büller for contributing to the design of the placebo-control study.
REFERENCES

1. Kearon C, Kahn SR, Agnelli G, Goldhaber S, Raskob GE, Comerota AJ. Antithrombotic therapy for venous thromboembolic disease. Chest 2008;133:Suppl: 454S-545S. [Erratum, Chest 2008;134:892.] 2. Ost D, Tepper J, Mihara H, Lander O, Heinzer R, Fein A. Duration of anticoagulation following venous thromboembolism: a meta-analysis. JAMA 2005;294:706-15.

3. Schulman S, Kearon C, Kakkar AK, et al. Dabigatran versus warfarin in the treatment of acute venous thromboembolism. N Engl J Med 2009;361:2342-52.

4. Schulman S, Kakkar AK, Schellong SM, et al. A randomized trial of dabigatran versus warfarin in the treatment of acute venous thromboembolism (RE-COVER II). Presented at the American Society of Hematology Annual Meeting, San Diego, CA, December 10-13, 2011. abstract.

5. Kearon C, Gent M, Hirsh J, et al. A comparison of three months of anticoagulation with extended anticoagulation for a first episode of idiopathic venous thromboembolism. N Engl J Med 1999; 340:901-7. [Erratum, N Engl J Med 1999; 341:298.]

6. Kearon C, Ginsberg JS, Kovacs MJ, et al. Comparison of low-intensity warfarin therapy with conventional-intensity warfarin therapy for long-term prevention of recurrent venous thromboembolism. N Engl J Med 2003;349:631-9.

7. Ridker PM, Goldhaber SZ, Danielson $\mathrm{E}$, et al. Long-term, low-intensity warfarin therapy for the prevention of recurrent venous thromboembolism. N Engl J Med 2003;348:1425-34.
8. Schulman S, Wåhlander $\mathrm{K}$, Lundström T, Clason SB, Eriksson H. Secondary prevention of venous thromboembolism with the oral direct thrombin inhibitor ximelagatran. N Engl J Med 2003;349: 1713-21.

9. Bauersachs R, Berkowitz SD, Brenner $B$, et al. Oral rivaroxaban for symptomatic venous thromboembolism. N Engl J Med 2010;363:2499-510.

10. Büller HR, Cohen AT, Davidson B, et al. Idraparinux versus standard therapy for venous thromboembolic disease. $\mathrm{N}$ Engl J Med 2007;357:1094-104.

11. Büller HR, Davidson BL, Decousus H, et al. Fondaparinux or enoxaparin for the initial treatment of symptomatic deep venous thrombosis: a randomized trial. Ann Intern Med 2004;140:867-73.

12. Idem. Subcutaneous fondaparinux versus intravenous unfractionated heparin in the initial treatment of pulmonary embolism. N Engl J Med 2003;349:1695-702. [Erratum, N Engl J Med 2004;350:423.]

13. Fiessinger JN, Huisman MV, Davidson $\mathrm{BL}$, et al. Ximelagatran vs low-molecularweight heparin and warfarin for the treatment of deep vein thrombosis: a randomized trial. JAMA 2005;293:681-9.

14. Kearon C, Ginsberg JS, Julian JA, et al. Comparison of fixed-dose weight-adjusted unfractionated heparin and lowmolecular-weight heparin for acute treatment of venous thromboembolism. JAMA 2006;296:935-42.

15. Hutten BA, Prins MH. Duration of treatment with vitamin $\mathrm{K}$ antagonists in symptomatic venous thromboembolism.
Cochrane Database Syst Rev 2000;3: CD001367.

16. Agnelli G, Prandoni P, Becattini C, et al. Extended oral anticoagulant therapy after a first episode of pulmonary embolism. Ann Intern Med 2003;139:19-25.

17. Agnelli G, Prandoni P, Santamaria MG, et al. Three months versus one year of oral anticoagulant therapy for idiopathic deep venous thrombosis. $\mathrm{N}$ Engl J Med 2001;345:165-9.

18. Schulman S, Granqvist S, Holmström $\mathrm{M}$, et al. The duration of oral anticoagulant therapy after a second episode of venous thromboembolism. N Engl J Med 1997;336:393-8.

19. Connolly SJ, Ezekowitz MD, Yusuf S, et al. Dabigatran versus warfarin in patients with atrial fibrillation. $\mathrm{N}$ Engl J Med 2009;361:1139-51. [Erratum, N Engl J Med 2010;363:1877.]

20. Connolly SJ, Ezekowitz MD, Yusuf S, Reilly PA, Wallentin L. Newly identified events in the RE-LY trial. N Engl J Med 2010;363:1875-6.

21. Hohnloser SH, Oldgren J, Yang S, et al. Myocardial ischemic events in patients with atrial fibrillation treated with dabigatran or warfarin in the RE-LY (Randomized Evaluation of Long-Term Anticoagulation Therapy) trial. Circulation 2012; 125:669-76.

22. Uchino K, Hernandez AV. Dabigatran association with higher risk of acute coronary events: meta-analysis of noninferiority randomized controlled trials. Arch Intern Med 2012;172:397-402.

Copyright (c) 2013 Massachusetts Medical Society. 